Fetal Diagnosis

and Therapy
Fetal Diagn Ther 2012;31:12-18

DOI: $\underline{10.1159 / 000331408}$
Received: June 22, 2011

Accepted after revision: July 27, 2011

Published online: December 14, 2011

\title{
Mid-Term Neurodevelopmental Outcome in Isolated Mild Ventriculomegaly Diagnosed in Fetal Life
}

\author{
Paula Gómez-Arriaga ${ }^{a} \quad$ Ignacio Herraiz ${ }^{\mathrm{a}}$ Jose Manuel Puente ${ }^{\mathrm{a}}$ \\ Berta Zamora-Crespo ${ }^{b}$ Noemi Núñez-Enamorado ${ }^{c}$ Alberto Galindo ${ }^{a}$

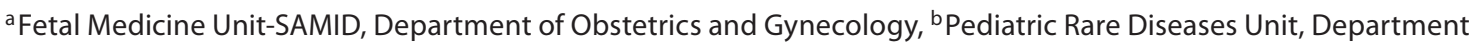 \\ of Pediatrics, and 'Pediatric Neurology Unit, Department of Pediatrics, Hospital Universitario '12 de Octubre', \\ Madrid, Spain
}

\section{Key Words}

Fetal mild ventriculomegaly $\cdot$ Neurodevelopmental outcome $\cdot$ Prenatal diagnosis $\cdot$ Battelle Developmental Inventory Screening Test

\begin{abstract}
Objective: To analyze mid-term neurodevelopment outcome in children with isolated mild ventriculomegaly (VM) $\leq 12 \mathrm{~mm}$ diagnosed in fetal life, using the Battelle Developmental Inventory Screening Test (BDIST). Methods: 86 cases of mild VM were identified. 68 were excluded due to: other cerebral anomalies $(n=40)$, extra-cerebral anomalies $(n=3)$, chromosomal defects $(n=4)$, dysmorphic syndromes $(n=4)$, congenital infections $(n=2)$, termination of pregnancy $(n=$ 9), stillbirth ( $n=2)$ and incomplete follow-up $(n=4) .18$ cases (range 1-8 years) of isolated mild VM were included for analysis. Seven neurodevelopment domains were assessed by BDIST. Results: Routine neuropediatrical evaluation detected neurological disorders in five children (28\%; 3 with language impairment, one left hemiparesis and one intellectual retardation). BDIST showed some degree of neurodevelopmental delay in higher proportions: $66 \%$ in social-personal skills, $56 \%$ in gross motor skills, $39 \%$ in adaptive behavior and $28 \%$ in fine motor skills. Communicative and cognitive areas
\end{abstract}

were the least affected (11 and 22\% had moderate-to-severe involvement, respectively). A general trend towards worse outcomes was observed in the group of $\geq 4$ years, although significant differences were only found for gross motor skills. Conclusion: Subtle neurological delays may appear during the infant period in fetuses prenatally diagnosed of isolated mild VM. In consequence, adequate measures should be established for early detection and treatment.

Copyright $\odot 2011$ S. Karger AG, Basel

\section{Introduction}

Ventriculomegaly (VM) is the most common anomaly of the central nervous system diagnosed in fetal life. The prevalence of VM varies between 0.3 and 1.5 per 1,000 births, depending on the technique used for measuring, the evaluation of one or both lateral ventricles and gestational age at examination [1]. This prevalence is 20 fold increased in high-risk populations [2]. Up to $50 \%$ of cases are associated with other abnormalities (structural defects 33-61\%, chromosomal aberrations 3-9\% and congenital infection 5\%) [3] and their detection is essential for proper prognosis assessment. This is more difficult when the VM presents in isolation. In fact, although

\section{KARGER}

Fax +41613061234 E-Mail karger@karger.ch www.karger.com

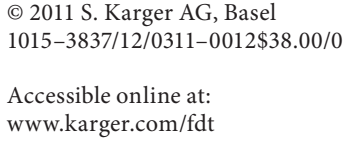

\section{Dr. A. Galindo}

Fetal Medicine Unit

Department of Obstetrics and Gynecology, Hospital Universitario '12 de Octubre' Avenida de Córdoba s/n, ES-28041 Madrid (Spain)

Tel. +34 913908 310, E-Mail agalindo.hdoc@ salud.madrid.org 
a relationship between the degree of ventricular dilatation and the neurological impact seems to have been established, the rate of psychomotor retardation in severe forms of VM ( $\geq 15 \mathrm{~mm}$ ) varies widely among series, between 37.5 and $89 \%[4,5]$.

Despite the fact that the majority of cases of fetal VM found in clinical practice fall into the mild category [6], the implications of isolated forms are poorly known, mainly due to the heterogeneity of the criteria used to define mild VM (10-15 vs. $10-12 \mathrm{~mm})[6,7]$. Moreover, there is currently little available information about mid and long-term outcome of children diagnosed prenatally of mild VM $[5,8]$ and, in addition, follow-up studies are often incomplete and subjective $[9,10]$. Therefore, prenatal assessment in these cases usually represents a conflictive situation both for parents and clinicians. This means that it is necessary to collect mid and long-term data on the neurological outcome of these children, in order to provide the parents the most accurate counseling following prenatal diagnosis of isolated mild VM. Furthermore, precocious identification of potential psychomotor deficits could allow an early implementation of effective intervention measures designed to minimize their impact on neurodevelopment.

The aim of this study is to analyze mid-term postnatal outcome in a cohort of infants with prenatally detected isolated mild VM analyzing the results provided both by the routine neuropediatric clinical assessment and by an additional structured interview to the parents.

\section{Methods}

\section{Prenatal Issues}

This is a retrospective study performed at a tertiary care referral center for prenatal diagnosis and management of fetal and neonatal pathology. The study was approved by the local ethics committee. Our database was queried for cases of mild VM prenatally diagnosed in the period from 2002 to 2008. Mild VM was defined as a transverse diameter of one or both ventricular atria between $>10.0$ and $\leq 12.0 \mathrm{~mm}$ [11]. All measurements of lateral ventricles were obtained according to current recommendations [12]. With these criteria, 86 cases were retrieved and analyzed.

Fetal neurosonography and a thorough sonographic evaluation of the rest of fetal morphology were performed in all cases by a fetal medicine specialist. All ongoing pregnancies underwent sonographic follow-up every 4-6 weeks in order to detect progression of the ventricular dilation and/or associated defects of late onset. Karyotype analysis was offered in all cases: in 46 cases it was performed by amniocentesis (54\%) and in 24 cases by fetal blood sampling (28\%). In the rest, the karyotype was studied postnatally according to neonatal clinical findings. In all cases of apparently isolated VM, TORCH analysis was performed. In two cases, maternal seroconversion to cytomegalovirus was detected during pregnancy, and subsequent polymerase chain reaction amplification of cytomegalovirus DNA in amniotic fluid sample confirmed fetal infection. From 2006, we also included fetal magnetic resonance imaging (MRI) after the initial diagnosis of apparently isolated mild VM by neurosonography, in order to confirm the diagnosis and exclude associated CNS anomalies. Ultrasound findings, test results and pregnancy outcomes were entered in the database as soon as they became available.

\section{Postnatal Issues}

A detailed neonatal examination of all patients included in the study was conducted in the first week of life, including general and neurological clinical assessment and a cerebral transfontanelar ultrasound (TF-US) in order to confirm the prenatal diagnosis. Postnatal MRI was indicated whenever there were concerns regarding the presence of associated defects after performing TFUS. All patients with isolated mild VM underwent clinical followup in an outpatient basis by a neuropediatrician specialist, according to the following policy: firstly, an additional TF-US was performed at the end of the first month of life in order to detect a possible progression of the VM. Secondly, a clinical evaluation including a complete physical and neurological examination, measurement of head circumference and psychomotor development assessment by checking if the chronological acquisition of the main neurological milestones are appropriated was performed at 1, 3, 6, 12, 18 and 24 months of age. Thirdly, TF-US was also performed in each visit as long as the fontanelles remained open. Finally, MRI was requested whenever there was progression of the VM or when the clinical examination revealed either psychomotor retardation or neurological deficits. If all these revisions displayed normal results, the infant was discharged from the neuropediatric office. Otherwise, the patient remained under specialized surveillance.

All cases in which cranial or extracranial associated defects, chromosomal abnormalities and/or congenital infections were detected either prenatally or postnatally were excluded. Similarly, terminated pregnancies, stillbirths and patients lost to follow-up were also excluded. Table 1 summarizes all cases of mild VM diagnosed in fetal life and reasons for exclusion from the study.

Information about neurological developmental status of patients included in the study was obtained consulting medical records. Psychomotor development was examined by a clinical psychologist using the Battelle Developmental Inventory Screening Test (BDIST). This questionnaire is the only test to examine pediatric neurodevelopment which has been validated to be applied as a screening tool in Spanish [13]. The BDIST has three administration formats (structured administration, observation, and interviews with parents) which can be used either in isolation or in a complementary manner to get the information [14]. Due to the fact that the majority of our patients were referred from distant areas of our country, we obtained all the data by means of telephone interview with the parents.

Briefly, BDIST is a widely used clinical tool for assessing key developmental skills in children from 6 months to 8 years of age. It is comprised of 96 age-specific items to evaluate the following five domains: personal-social skills, adaptive behavior, psychomotor ability (including fine motor and gross motor skills), communication, and cognition. Mean and standard deviations (SD) from the scores in each domain are calculated to classify patients either as normal, borderline ( $<1$ SD below the mean), mild retar- 
Table 1. Summary of the fetuses diagnosed with mild ventriculomegaly that were excluded from further analysis (each fetus has been included only once, under the most important category)

\begin{tabular}{ll}
\hline Causes of exclusion & $\mathrm{n}(\%)$ \\
\hline Central nervous system anomalies & $40(58.8)$ \\
Spina bifida/myelomeningocele & 13 \\
Agenesis of the corpus callosum & 13 \\
Intrauterine progression to severe ventriculomegaly & 3 \\
Cerebral hemorrhage & 3 \\
Dandy-Walker syndrome & 2 \\
Arnold-Chiari malformation & 2 \\
Heterotopia* & 2 \\
Schizencephaly* & 1 \\
Subarachnoid cyst & 1 \\
Non-central nervous system anomalies & $3(4.4)$ \\
Urinary tract anomalies & 1 \\
$\quad$ Bilateral hydronephrosis & 1 \\
Non-immune hydrops fetalis & 1 \\
Congenital heart disease & \\
$\quad$ Ventricular septal defect & $4(5.8)$ \\
Chromosomal defects & 1 \\
69,XXX & 1 \\
46,XX,-8, +der(8)t(8;15)(p22;q23) & 1 \\
47,XX, +18 & 1 \\
46,XX,del(8)(p21.2;pter) & $4(5.8)$ \\
Dysmorphic non-chromosomal syndromes & $2(2.9)$ \\
Congenital infections & 2 \\
Cytomegalovirus & 98 \\
Terminations of pregnancy & $2(2.9)$ \\
Stillbirths & $4(5.8)$ \\
Incomplete postnatal follow-up & 68 \\
\hline Total & \\
\hline &
\end{tabular}

* Diagnosed in postnatal MRI.
Table 2. Summary of the characteristics of the cases of mild fetal ventriculomegaly diagnosed prenatally

\begin{tabular}{ll}
\hline Variable & Value \\
\hline $\begin{array}{l}\text { Number of mild-to-moderate isolated } \\
\quad \text { ventriculomegaly prenatally diagnosed }\end{array}$ & \\
$\quad$ with complete follow-up & 18 \\
Mean maternal age, years (range) & $29(20-38)$ \\
Mean gestational age at diagnosis, weeks (range) & $22(19-33)$ \\
Diagnosis $\leq 22$ weeks* $(\%)$ & $13(72.2)$ \\
\hline Characteristics of prenatal diagnosis & \\
Women referred from their local hospitals & \\
$\quad$ after the finding of fetal ventriculomegaly & \\
$\quad$ on a routine ultrasound scan (\%) & $10(55.5)$ \\
Women primarily attending our hospital & \\
$\quad$ in which the diagnosis was made on a & \\
$\quad$ routine ultrasound scan (\%) & $8(44.5)$ \\
\hline $\begin{array}{l}\text { Isolated ventriculomegaly } \\
\text { Unilateral (\%) }\end{array}$ & \\
Bilateral (\%) & \\
Mean (SD) size \pm SD of the posterior horns of & $13(27.8)$ \\
$\quad$ lateral ventricles, mm & $10.95(1.05)$ \\
Male/female & $11 / 7$ \\
Spontaneous resolution (\%) & $3(16.6)$ \\
Mean gestational age at birth, weeks (range) & $38(34-41)$ \\
$\quad$ Preterm delivery $\leq 37$ weeks (\%) & $4(22.2)$ \\
\hline &
\end{tabular}

* Upper limit for termination of pregnancy in our country. $\dagger$ The policy in our country regarding antenatal care in low-risk pregnancies includes the routine performance of an obstetric ultrasound scan in the third trimester, usually between 28 and 34 weeks, to assess fetal growth and well-being, and to detect congenital defects of late-onset and/or those which might be overlooked in the mid-second trimester scan.

$\mathrm{SD}=$ standard deviation;

\section{Results}

The study group included 18 liveborn fetuses that fulfilled all the inclusion criteria. This figure represents $21 \%$ of all mild VM prenatally diagnosed during the study period. All cases were incidental findings in low-risk pregnancies at the routine second or third trimester ultrasound scans. Their most important clinical data are summarized in table 2. At least one additional ultrasound examination was performed in all cases after the initial prenatal diagnosis, and the majority $(15 / 18,83 \%)$ had at least two additional scans. In these follow-up scans, the ventricular dilation remained stable in all fetuses. Postnatally, only one patient showed to have a progressive VM which became severe at 12 months of age and required ventriculo-peritoneal shunting. 
Table 3. Distribution of the results of the Battelle Developmental Inventory Screening Test in our series of 18 infants prenatally diagnosed with isolated mild ventriculomegaly

\begin{tabular}{lrlll}
\hline \multirow{2}{*}{ Area } & Normal, n (\%) & Abnormal, n (\%) & \\
\cline { 4 - 5 } & & borderline <1 SD & moderate <1-2 SD & severe <2 SD \\
\hline Personal-social & $6(33.3)$ & $6(33.3)$ & $4(22.2)$ & $2(11.1)$ \\
Adaptive & $11(61.1)$ & $2(11.1)$ & $1(5.5)$ & $4(22.2)$ \\
Gross motor skills & $8(43.5)$ & $6(33.3)$ & 0 & $4(22.2)$ \\
Fine motor skills & $13(72.2)$ & 0 & $2(11.1)$ & $3(16.7)$ \\
Receptive communication & $11(61.1)$ & $4(22.2)$ & $1(5.5)$ & $2(11.1)$ \\
Expressive communication & $14(77.8)$ & 0 & 0 & $4(22.2)$ \\
Cognition & $15(83.3)$ & $1(5.5)$ & 0 & $2(11.1)$ \\
Overall development & $14(77.8)$ & 0 & $3(16.7)$ & $1(5.5)$ \\
\hline
\end{tabular}

$\mathrm{SD}=$ Standard deviation

Postnatal neurologic follow-up showed a completely normal outcome in most infants $(13 / 18,72.2 \%)$. Neurological disorders were detected in 5 patients, including 3 with mild-to-moderate language impairment requiring a speech therapist, 1 with a left hemiparesis mainly affecting the left hand and which was successfully treated with botulinum toxin and the infant with progressive VM, who suffers from severe intellectual retardation requiring supportive measures.

The mean age of the infants at the time of the neurodevelopment evaluation with BDIST was 4.9 years (range $1-8)$. BDIST showed some degree of psychomotor delay in a higher proportion of children than the routine clinical neurologic follow-up (table 3).

Disabilities affecting social-personal skills, which includes interaction with adults, expression of emotions, self-concept, peer interaction, and social role, were reported in two thirds of the patients, and in half of the cases this delay was moderate to severe. Similarly, a high proportion of disorders was also found in the adaptive behavior domain $(7 / 18,39 \%)$, which includes attention, eating, dressing, toileting and personal responsibility. One third of the infants $\geq 4$ years showed to have a severe adaptive behavior disorder. Furthermore, a high rate of alterations was detected in the 'gross motor skills' section (those affecting the ability of children to carry out activities that require large muscles or groups of muscles) $(10 / 18,56 \%)$, although the $60 \%$ of them were minor deviations from normality. 'Fine motor skills' disabilities (those affecting the movements of small muscles that act in an organized and subtle fashion to accomplish delicate tasks) were less common $(5 / 18,28 \%)$, but all cases suffered from a moderate-to-severe impairment. Communicative and cognitive areas were the least affected and moderateto-severe forms of involvement were reported in only 11 and $22 \%$ of our patients, respectively.

When comparing the proportion of abnormal findings in each domain between the group of children in whom the BDIST was applied before and after 4 years of age, a general trend towards worse outcomes can be observed in the group of $\geq 4$ years. However, significant differences were only found in the 'gross motor skills' area (fig. 1).

We performed a further subanalysis to examine the relationship between the overall development according to BDIST and prognostic variables as gestational age at diagnosis (<22 weeks vs. $\geq 22$ weeks), fetal sex (male vs. female) and laterality (unilateral vs. bilateral VM). This subanalysis showed no significant differences for these variable ( $p$ values: $0.65,0.71$ and 1.00 , respectively), but in all cases diagnosed after 22 weeks (5/5), the overall development was inside the normal range.

\section{Discussion}

Prenatal diagnosis of isolated mild VM represents a considerable challenge when counseling about neurological outcome. Firstly, parents should be advised that this is a provisional diagnosis until associated malformations, congenital infections and chromosomal defects can be excluded. Fortunately, these abnormalities can be rule out prenatally in most cases. The main dilemma relies on the fact that it is not possible to distinguish with certainty which fetuses with isolated mild VM will develop neurological sequelaes postnatally. In this sense, fetal MRI 


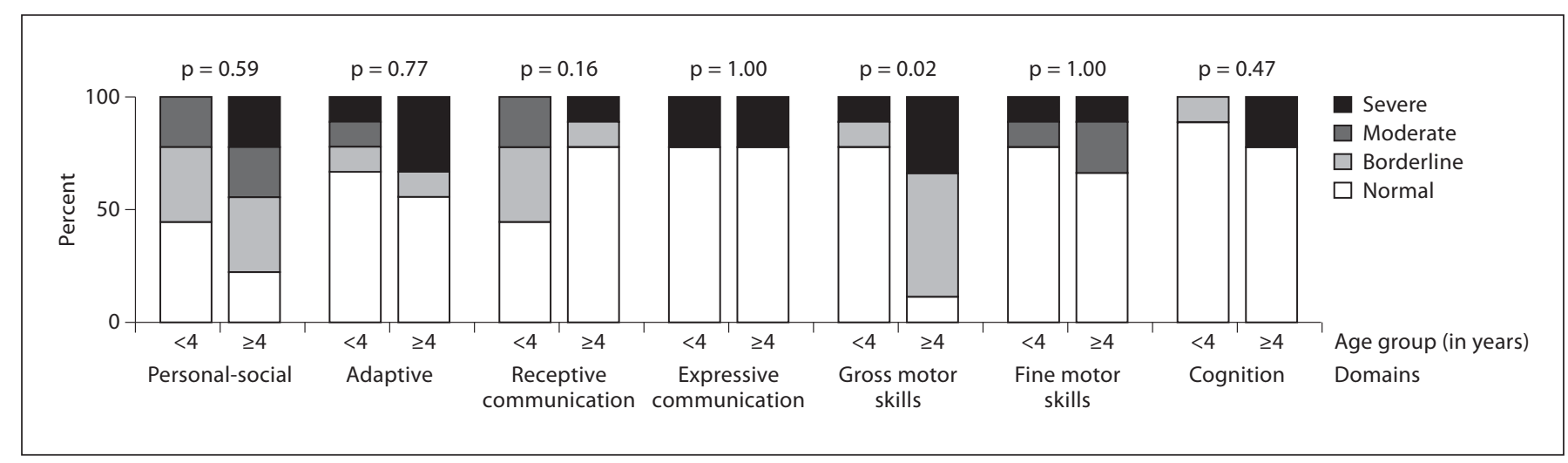

Fig. 1. Distribution by group of age ( $<4$ years vs. $\geq 4$ years) of neurodevelopmental outcomes in the seven domains assessed by the Battelle Developmental Inventory Screening Test (BDIST).

for further evaluation of isolated mild VM in neurosonography has not been proved to add relevant information beyond the diagnostic confirmation, but this may be important to reassure the parents, specifically in the midsecond trimester of pregnancy. Notwithstanding, it is of paramount importance to provide the parents with the most accurate counseling about the risks of neurological impairment in their child.

Several studies have analyzed the neurological outcome of children with a prenatal diagnosis of isolated mild VM, but there is a wide variation in the reported results $[4,5]$. These discrepancies are mainly due to heterogeneity in the definition of mild VM, the use of different methods of neurodevelopment assessment and the lack of distinction on the degree of delay [10]. Few studies have conducted mid or long-term follow-up using objective tests to assess the severity of delay in distinct areas of neurodevelopment. Our study restricted the diagnosis of mild VM to measurements of $10-12 \mathrm{~mm}$, representing a spectrum of cases very close to the threshold of $10 \mathrm{~mm}$, below which the ventricular measurement is considered normal by consensus [16]. It has been stated that ventricles between $>12$ and $\leq 15 \mathrm{~mm}$ should be studied separately as they are related to more unfavorable outcomes [17]. Although it has even been suggested that lateral ventricular atrium between 10 and $12 \mathrm{~mm}$ should be considered a variant of the norm [6], the postnatal neurologic follow-up of our cases revealed abnormal findings in $28 \%$ and intellective retardation in one child (5\%). Our results correlate well with previous reports [18] and with a recent review which has reported abnormal neurological outcome in 34/288 (12\%) of chromosomally normal infants presenting antenatally with an atrial width between 10 and $11.9 \mathrm{~mm}$ [7]. Therefore, this collection of results further demonstrates that isolated mild VM should be considered as a separate entity.

In addition, we explored the presence of subtle alterations in the psychomotor development of these children, which may be unnoticed by most parents who do not possess tools to detect non-obvious difficulties, and by pediatricians, who may not be able to observe them in a routine clinical examination. For that purpose, we applied a developmental screening test, as this tool has shown to improve the accuracy for identifying developmental delays in comparison with routine neuropediatric clinical evaluation. Certainly, the sensitivity and specificity of these screening instruments are usually reported between 70 and $90 \%$ [19].

Of all the tests available, we selected the BDIST for two main reasons. Firstly, it is a widely validated screening test in all its administration formats to be used in children with a wide range of ages between 6 months and 8 years [20], which fitted well with the ages of our patients. Secondly, this test allowed us to inquire into the parental perception about their child, without requiring moving the family from their diverse points of origin. Although we could not conduct a direct observation of the children, parents' responses were sufficient to complete the test in all cases. Obviously, the scores obtained in this manner are merely indicative of the potential existence of neurodevelopmental delays, and the clinical significance of this information should be established using specific diagnostic tests applied by an expert.

Using this approach, we find out that the most common disabilities in these patients affect the gross motor skills. This is in accordance with two previous reports 
[21-23], although a definitive explanation for this finding remains elusive [11]. It is noteworthy that the degree of impairment was significantly greater with advancing age. It may have practical implications since the prognosis of these children could be improved by strengthening specific exercises from early infancy, when the deficiency is still not evident, in order to prevent the onset and/or progression of damage in motor areas [19, 24, 25].

Another interesting result of our study was the surprisingly high frequency of problems found in the personal-social and behavior areas. This could be explained, in part, by an attitude of parental overprotection of these children, which interferes with their social integration. A similar phenomenon has been described in children who have been stigmatized with other diagnosis, such as congenital heart disease [26], spina bifida [27] and corpus callosum agenesis [28]. The fact that isolated mild VM does not usually affect the intellectual development, may influence parents and pediatricians not to notice the need for early and active stimulation in other areas of neurological development, allowing these deficits become more evident over time and with increasing academic demands on these children.

We acknowledge three limitations in our study. Firstly, the retrospective nature of the study, but it was based on detailed collected data. Secondly, the sample size was relatively small, a problem also found in previous series. This shortcoming may limit the strength of our conclusions. However, it is not easy to recruit ongoing pregnancies with truly isolated mild VM, given that fetal VM is associated with other abnormalities in more than $50 \%$ of the cases [3], and even after excluding associated defects, $34 \%$ of parents choose termination of pregnancy due to concerns regarding neurological outcome [29]. This limited number of cases also precluded us to draw firm statements about the significance of prognostic factors such as gestational age at diagnosis, fetal sex and symmetry of VM on neurological development. Finally, a part of our results are based on a development screening test conducted on parents, so further studies are needed to confirm these results through a full assessment. Such assessment requires costly resources and highly skilled professionals, and it is beyond the scope of our study. However, parents' concerns have showed to provide accurate information about their child's development [19], and we believe that they represent an essential view to communicate to other parents the degree of involvement which may be associated with mild VM.

In conclusion, isolated mild VM $(10-12 \mathrm{~mm})$ in the prenatal period constitutes an easy-to-detect diagnosis but a difficult-to-assess condition. After excluding other associated abnormalities, information about postnatal outcome should be fundamentally reassurance as the risk of severe cognitive retardation is only about $5 \%$. However, parents and pediatricians should be advised that mild-to-moderate delays in different areas of neurological development may appear during the infant period. Therefore, adequate screening strategies should be implemented to maximize the options of early detection of these delays, in order to establish early supportive measures and minimize their impact in the developing child.

\section{References}

1 Pilu G, Hobbins J: Sonography of fetal cerebrospinal anomalies. Prenat Diagn 2002;22: 321-330.

$\checkmark 2$ Achiron R, Schimmel M, Achiron A, Mashiach S: Fetal mild idiopathic lateral ventriculomegaly: is there a correlation with fetal trisomy? Ultrasound Obstet Gynecol 1993;3:89-92.

-3 Kelly EN, Allen VM, Seaward G, Windrim R, Ryan G: Mild ventriculomegaly in the fetus, natural history, associated findings and outcome of isolated mild ventriculomegaly: a literature review. Prenat Diagn 2001;21:697-700.

-4 Gaglioti P, Danelon D, Bontempo S, Mombrò M, Cardaropoli S, Todros T: Fetal cerebral ventriculomegaly: outcome in 176 cases. Ultrasound Obstet Gynecol 2005;25:372-377.

5 Graham E, Duhl A, Ural S, Allen M, Blakemore K, Witter F: The degree of antenatal ventriculomegaly is related to pediatric neurological morbidity. J Matern Fetal Med 2001;10:258-263.

6 Signorelli M, Tiberti A, Valseriati D, Molin E, Cerri V, Groli C, Bianchi UA: Width of the fetal lateral ventricular atrium between 10 and $12 \mathrm{~mm}$ : a simple variation of the norm? Ultrasound Obstet Gynecol 2004;23:14-18.

7 Melchiorre K, Bhide A, Gika AD, Pilu G, Papageorghiou AT: Counseling in isolated mild fetal ventriculomegaly. Ultrasound Obstet Gynecol 2009;34:212-224.

8 Falip C, Blanc N, Maes E, Zaccaria I, Oury JF, Sebag G, Garel C: Postnatal clinical and imaging follow-up of infants with prenatal isolated mild ventriculomegaly: a series of 101 cases. Pediatr Radiol 2007;37:981-989.

$\checkmark 9$ Vergani P, Locatelli A, Strobelt N, Cavallone M, Ceruti P, Paterlini G, Ghidini A: Clinical outcome of mild fetal ventriculomegaly. Am J Obstet Gynecol 1998;178:218-222.

$\checkmark 10$ Melchiorre K, Liberati M, Celentano C, Domizio S, Puglielli C, Buoni S, Strambi M, Zannolli R: Neurological outcome following isolated $10-12 \mathrm{~mm}$ fetal ventriculomegaly. Arch Dis Child Fetal Neonatal Ed 2009; 94:F311-F312.

11 Gaglioti P, Oberto M, Todros T: The significance of fetal ventriculomegaly: etiology, short- and long-term outcomes. Prenat Diagn 2009;29:381-388.

-12 International Society of Ultrasound in Obstetrics and Gynecology. Sonographic examination of the fetal central nervous system: guidelines for performing the 'basic examination' and the 'fetal neurosonogram'. Ultrasound Obstet Gynecol 2007;29:109116. 
13 Newborg J, Stock JR, Wnek L: Batelle, inventario de desarrollo. Madrid, TEA, 2004.

- 14 Berls AT; McEwen IR: Battelle Developmental Inventory. Phys Ther 1999;79:776-783.

15 Newborg J, Stock JR, Wnek L, Guidubaldi J, Svinicki J: The Battelle Developmental Inventory. Allen, TX: DLM Teaching Resources; 1988.

16 Salomon LJ, Bernard JP, Ville Y: Reference ranges for fetal ventricular width: a non-normal approach. Ultrasound Obstet Gynecol 2007;30:61-66

-17 Arora A, Bannister CM, Russell S, Rimmer $S$ : Outcome and clinical course of prenatally diagnosed cerebral ventriculomegaly. Eur J Pediatr Surg 1998;8(suppl 1):63-64.

-18 Pilu G, Falco P, Gabrielli S, Perolo A, Sandri F, Bovicelli L: The clinical significance of fetal isolated cerebral borderline ventriculomegaly: report of 31 cases and review of the literature. Ultrasound Obstet Gynecol 1999; 14:320-326.

-19 Rydz D, Srour M, Oskoui M, Marget N, Shiller M, Birnbaum R, Majnemer A, Shevell MI: Screening for developmental delay in the setting of a community pediatric clinic: a prospective assessment of parent-report questionnaires. Pediatrics 2006;118:e1178e1186.
20 Glascoe FP, Byrne KE: The usefulness of the Battelle Developmental Inventory Screening Test. Clin Pediatr (Phila) 1993;32:273-280.

21 Beeghly M, Ware J, Soul J, du Plessis A, Khwaja O, Senapati GM, Robson CD, Robertson RL, Poussaint TY, Barnewolt CE, Feldman HA, Estroff JA, Levine D: Neurodevelopmental outcome of fetuses referred for ventriculomegaly. Ultrasound Obstet Gynecol 2010;35:405-416.

22 Bloom SL, Bloom DD, DellaNebbia C, Martin LB, Lucas MJ, Twickler DM: The developmental outcome of children with antenatal mild isolated ventriculomegaly. Obstet Gynecol 1997;90:93-97.

-23 Sadan S, Malinger G, Schweiger A, Lev D, Lerman-Sagie T: Neuropsychological outcome of children with asymmetric ventricles or unilateral mild ventriculomegaly identified in utero. Br J Obstet Gynaecol 2007;114: 596-602.
24 Reynolds AJ, Temple JA, Robertson DL, Mann EA: Long-term effects of an early childhood intervention on educational achievement and juvenile arrest: A 15-year follow-up of low-income children in public schools. JAMA 2001;285:2339-2346.

25 Riethmuller AM, Jones R, Okely AD: Efficacy of interventions to improve motor development in young children: a systematic review. Pediatrics 2009;124:e782-e792.

26 Ong L, Nolan RP, Irvine J, Kovacs AH: Parental overprotection and heart-focused anxiety in adults with congenital heart disease. Int J Behav Med 2010, DOI: 10.1007/ s12529-010-9112-y.

27 Vermaes IP, Gerris JR, Janssens JM: Parents' social adjustment in families of children with spina bifida: a theory-driven review. J Pediatr Psychol 2007;32:1214-1226.

28 Chiappedi M, Bejor M: Corpus callosum agenesis and rehabilitative treatment. Ital J Pediatr 2010;36:64

-29 Joó JG, Tóth Z, Beke A, Papp C, Tóth-Pál E, Csaba A, Szigeti Z, Rab A, Papp Z: Etiology, prenatal diagnostics and outcome of ventriculomegaly in 230 cases. Fetal Diagn Ther 2008;24:254-263. 